\section{Bioimpedância elétrica e gordura visceral: uma comparação com a tomografia computadorizada em adultos e idosos}

\author{
Bioelectrical impedance and visceral fat: a comparison \\ with computed tomography in adults and elderly
}

Michaela Eickemberg', Carolina Cunha de Oliveira²,

Anna Karla Carneiro Roriz ${ }^{3}$, Gardênia Abreu Vieira Fontes ${ }^{4}$,

Adriana Lima Mello5, Lílian Ramos Sampaio ${ }^{6}$

\begin{abstract}
RESUMO
Objetivo: Avaliar a associação entre bioimpedância elétrica (BIA) e gordura visceral (GV) em adultos e idosos. Sujeitos e métodos: Estudo transversal, 191 indivíduos (52\% mulheres, 49\% idosos), estratificados por sexo, grupo etário e massa corporal. Obtiveram-se dados sobre tomografia computadorizada (área de GV) e BIA (percentual de gordura corporal total (\%GCT-BIA), ângulo de fase, reactância e resistência). Análise estatística: Coeficiente de Correlação de Pearson, Anova, Qui-quadrado de Pearson, Curva ROC. Resultados: Áreas de GV $\geq 130 \mathrm{~cm}^{2}$ foram mais observadas em idosos e em homens. Entre as mulheres adultas, mostrou-se correlação mais forte entre GV e \%GCT-BIA. Os demais grupos apresentaram resultados semelhantes e correlações estatisticamente significantes. As correlações entre GV e ângulo de fase foram fracas e sem significância estatística. As análises da Curva ROC indicaram os seguintes \%GCT-BIA que identificaram excesso de GV: homens: 21,5\% (adultos), 24,25\% (idosos); mulheres: 35,05\% (adultas), 38,45\% (idosas), com sensibilidade de $78,6 \%, 82,1 \%, 83,3 \%, 66,7 \%$ e especificidade de $70,6 \%, 62,5 \%, 79,1 \%, 69 \%$, respectivamente. Conclusão: BIA apresentou satisfatória sensibilidade e especificidade para predizer GV, entretanto, outros aparelhos e técnicas devem ser investigados para melhorar essa predição. Arq Bras Endocrinol Metab. 2013;57(1):27-32
\end{abstract}

Descritores

Gordura visceral; impedância elétrica; tomografia computadorizada

\begin{abstract}
Objective: To evaluate the association between electrical bioimpedance analysis (BIA) and visceral fat (VF) in adult and elderly patients. Subjects and methods: This was a cross-sectional study, with a sample of 191 subjects ( $52 \%$ women, $49 \%$ elderly) stratified by sex, age and body mass. Computerized tomography (VF area) and BIA (percentage of total body fat (\%TBF-BIA), phase angle, reactance and resistance) data were generated. Statistical analysis was based on Pearson's Correlation Coefficient, Anova, Pearson's Chi-square, and ROC curves. Results: VF areas $\geq 130 \mathrm{~cm}^{2}$ were more prevalent among the elderly and among men. Adult females showed a stronger correlation between GV and \%TBF-BIA. The other groups showed similar results and statistically significant correlations. Correlations between GV and phase angle were weak and not statistically significant. ROC Curves analyzes showed the following \%TBF-BIA, which identified excess VF: for male subjects: 21.5\% (adults) and $24.25 \%$ (elderly); for female subjects: $35.05 \%$ (adults) and $38.45 \%$ (elderly) with sensitivity of $78.6 \%, 82.1 \%, 83.3 \%$, and $66.7 \%$, and specificity of $70.6 \%, 62.5 \%, 79.1 \%$, and $69 \%$, respectively. Conclusion: BIA was found to have satisfactory sensitivity and specificity to predict VF; however, other devices and other techniques should be investigated to improve VF prediction. Arq Bras Endocrinol Metab. 2013;57(1):27-32
\end{abstract}

Keywords

Visceral fat; electric impedance; computerized tomography
'Universidade Federal da Bahia (UFBA); Centro de Estudos e Intervenção na Área do Envelhecimento, Conselho Nacional de Desenvolvimento Científico e Tecnológico (CEIAECNPq), Salvador, BA, Brasil ${ }^{2}$ UFBA; Universidade Federal do Sergipe; CEIAE-CNPq, Salvador, BA, Brasil

${ }^{3}$ Departamento de Ciência da Nutrição, Escola de Nutrição, UFBA; CEIAE-CNPq, Salvador, BA, Brasil ${ }^{4}$ UFBA; Departamento de Ciência da Nutrição, Escola de Nutrição, UFBA, Salvador, BA, Brasil ${ }^{5}$ Universidade de São Paulo (USP), São Paulo, SP; Departamento de Ciência da Nutrição, Escola de Nutrição, UFBA; CEIAECNPq, Salvador, BA, Brasil

${ }^{6}$ Universidade Federal de São Paulo (Unifesp), São Paulo, SP Departamento de Ciência da Nutrição, Escola de Nutrição, UFBA; CEIAE-CNPq, Salvador, BA, Brasil

Estudo realizado no Hospital Universitário Professor Edgar Santos, UFBA, Salvador, BA

Correspondência para: Michaela Eickemberg Escola de Nutrição, Universidade Federal da Bahia Av. Araújo Pinho, 32 40110-150 - Salvador, BA, Brasil mieickemberg@yahoo.com.br

Recebido em 28/Fev/2012 Aceito em 4/Out/2012 


\section{INTRODUÇÃO}

A quantificação da gordura visceral (GV) é importante para avaliar o risco para o desenvolvimento de doenças crônicas não transmissíveis, dada a relação existente entre GV e doenças cardiovasculares $(1,2)$.

A tomografia computadorizada (TC) é um método considerado padrão-ouro para a detecção de GV, porém, tem elevado custo, necessita de técnico habilitado e a operacionalização é complexa $(3,4)$. A bioimpedância (BIA) é um método que avalia a composição corporal, estimando os compartimentos de gordura, massa muscular e hídrico, entretanto, estudos são necessários para avaliar se a GV é estimada com boa sensibilidade e especificidade pela BIA (5-7). Assim, a BIA se apresenta como um método alternativo, podendo este ser utilizado na prática clínica e epidemiológica.

Considerando a importância da GV como fator determinante de alterações metabólicas associadas à obesidade, a substituição da TC por um método mais simples, de baixo custo e livre de irradiação, significa um avanço no diagnóstico da obesidade visceral. E, amparado nessas convicções, este estudo objetiva avaliar a associação entre BIA e GV comparado à TC em adultos e idosos.

\section{SUJEITOS E MÉTODOS}

Trata-se de um estudo de corte transversal, realizado pela Escola de Nutrição da Universidade Federal da Bahia. Um total de 191 indivíduos, vinculados e não às Unidades Ambulatoriais de Saúde da Universidade Federal da Bahia, pertencentes a instituições geriátricas, participantes de projetos de outras Universidades e indivíduos da comunidade geral de Salvador foram selecionados randomicamente seguindo a estratificação por sexo, idade e massa corporal (avaliada pelo índice de massa corporal) como critérios de inclusão, conforme metodologia publicada em Roriz e cols. (8). Essa estratificação teve como intuito garantir a equivalência na representatividade dos grupos em relação à quantidade de gordura visceral que poderia estar associada a patologias.

Foram coletadas informações referentes às características demográficas, de BIA e de TC por meio de questionário e técnicas padronizados, aplicados por equipe devidamente treinada.

Não foram incluídos neste estudo os indivíduos com idade inferior a 20 anos, com IMC superior a 40 $\mathrm{kg} / \mathrm{m}^{2}$ ou com qualquer problema que comprometesse a verificação das medidas antropométricas e a acurada estimativa da gordura abdominal, como portadores de distúrbios graves, indivíduos que realizaram cirurgias abdominais recentes, amputados, gestantes ou mulheres que tiveram parto nos últimos seis meses, portadores de lesões e tumorações abdominais, hepatomegalia, esplenomegalia e ascite.

O estudo foi aprovado pela Comissão de Ética em Pesquisa da Escola de Nutrição da UFBA (Parecer $n^{\circ}$ 01/09) e todos os participantes assinaram o Termo de Consentimento Livre e Esclarecido.

\section{Bioimpedância elétrica}

Utilizou-se o aparelho tradicional marca Biodynamics modelo 310. O teste foi realizado com o indivíduo deitado sobre uma superfície não condutora, na posição supina, com braços e pernas abduzidos a $45^{\circ}$. Os voluntários foram orientados a seguir alguns procedimentos prévios: jejum absoluto de oito horas; não realizar exercícios físicos extenuantes nas 12 horas anteriores ao teste; não ingerir álcool 48 horas antes do teste; esvaziar a bexiga pelo menos 30 minutos antes da avaliação e retirar objetos metálicos do local de colocação dos eletrodos no momento da realização do teste $(9,10)$. As variáveis analisadas pela BIA foram: o percentual de gordura corporal total (\%GCT), reactância, resistência e ângulo de fase $(\mathrm{AF})$. O AF foi calculado por meio da fórmula: Reactância/Resistência x $180^{\circ} / \pi(11,12)$ para comparação com a GV medida pela TC. Esse parâmetro mede dano celular e avaliou-se sua relação com a GV e, consequentemente, com risco para desenvolver morbidades.

\section{Tomografia computadorizada}

A área de GV foi obtida por meio do tomógrafo Spirit Siemens, realizada por um mesmo técnico de radiologia e analisada por um mesmo observador. $\mathrm{O}$ exame foi realizado em jejum completo de quatro horas com o paciente em decúbito dorsal e os braços estendidos acima da cabeça. O corte tomográfico foi obtido com parâmetros radiográficos de $140 \mathrm{kV}$ e $45 \mathrm{~mA}$, no nível de L4L5, tendo espessura de $10 \mathrm{~mm}$ com tempo de exposição de três segundos. A área de GV foi determinada com cursor livre, tomando como limites as bordas internas dos músculos reto abdominal, oblíquo interno e quadrado lombar, excluindo-se o corpo vertebral e incluindo a gordura retroperitonial, mesentérica e omental. A área de gordura foi descrita $\mathrm{em} \mathrm{cm}^{2}$. Para identificação do tecido adiposo, utilizaram-se os valores de densidade de -50 e - 150 Unidades Hounsfields (13). Para indicar o excesso de GV (obesidade visceral), foi considerada uma área maior ou igual a $130 \mathrm{~cm}^{2}$ como risco para o desenvolvimento de doenças cardiovasculares (DCV) (14). 


\section{Análise estatística}

Por meio do programa SPSS Versão 11.5, os dados foram apresentados como média e amplitude de variação. Para avaliar a correlação entre as variáveis, foi utilizado o Coeficiente de Pearson. Foram calculadas as prevalências de obesidade geral e visceral. Para analisar a diferença de médias e a associação entre as variáveis relacionadas ao sexo e grupo etário foram utilizados os Testes Anova e Qui-quadrado de Pearson. A Receiver Operating Characteristic Curve - Curva ROC - foi construída usando um ponto de corte para o teste de referência, ou seja, a área de GV medida pela TC, onde foi selecionado o valor de $130 \mathrm{~cm}^{2}$ (teste de referência positivo). Para avaliar a performance da BIA, foram estimadas a sensibilidade e a especificidade de cada ponto de corte do \%GCT. O ponto de corte que produziu a melhor combinação de sensibilidade e especificidade foi selecionado como valor mais apropriado para a BIA predizer GV em excesso $\left(\geq 130 \mathrm{~cm}^{2}\right)$, para sexo e grupo etário.

\section{RESULTADOS}

A amostra foi de 191 indivíduos, sendo $48 \%$ do sexo masculino e $52 \%$ feminino e, segundo os grupos etários, $51 \%$ adultos e $49 \%$ idosos. As prevalências de obesidade geral, identificadas pelo IMC, para os homens foram $54,2 \%$ e $40,9 \%$ e, para as mulheres, $51 \%$ e $42 \%$, para adultos e idosos, respectivamente.

Com a finalidade de caracterizar a amostra, estão apresentados na tabela 1 os valores médios, mínimos e máximos da idade e IMC, além das variáveis obtidas por intermédio dos dois métodos analisados (BIA e TC), para sexo e grupo etário.
Os valores médios do IMC não apresentaram diferença significativa entre os grupos. Com relação às variáveis determinadas pelo método da BIA, observou-se que os idosos apresentaram valores médios de \%GCT acima dos valores dos adultos, e para as mulheres esse valor foi ainda maior $(37,2 \%)$. Os valores médios de reactância foram mais elevados nos adultos e, como consequência, o AF apresentou valores mais elevados neste grupo, quando comparado com o dos idosos.

Com relação à área de GV, medida por meio da TC, foram encontrados maiores valores médios entre os idosos de ambos os sexos. E, na comparação entre sexos, os homens apresentaram maiores valores, pertencendo ao grupo dos homens idosos as médias mais elevadas e superiores ao valor considerado como excesso de GV.

Conforme ilustra a figura 1 , as áreas de GV consideradas de risco $\left(\geq 130 \mathrm{~cm}^{2}\right)$ apresentaram maior prevalência no grupo dos idosos de ambos os sexos e, na análise por sexo, em homens. A mulher adulta representou o grupo de menor risco (12,20\%).

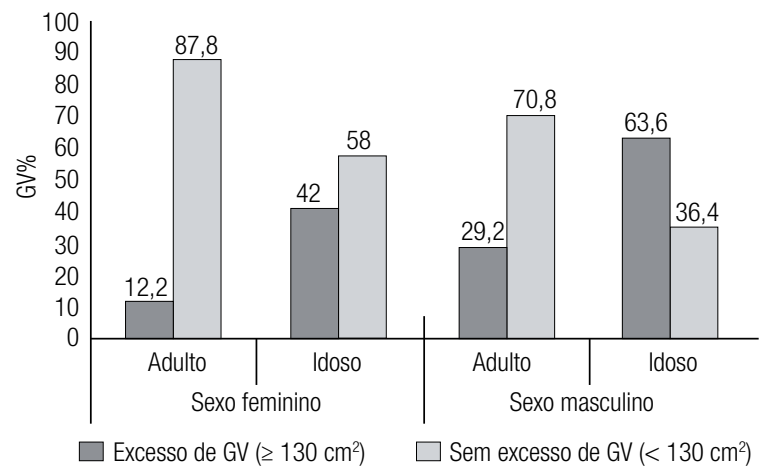

GV: gordura visceral; TC: tomografia computadorizada; \%GV: percentual de gordura visceral dado pela tomografia computadorizada.

Figura 1. Excesso de GV identificado pela TC, segundo sexo e grupo etário.

Tabela 1. Valores médios, mínimos e máximos da idade, índice de massa corporal e das variáveis obtidas por meio da BIA e TC, por sexo e grupo etário

\begin{tabular}{|c|c|c|c|c|}
\hline & \multicolumn{2}{|c|}{ Feminino } & \multicolumn{2}{|c|}{ Masculino } \\
\hline & Adulto & Idoso & Adulto & Idoso \\
\hline Idade & $39,94(21-58)^{1}$ & $73,70(60-90)^{1}$ & $39,37(21-59)^{1}$ & $72,00(60-95)^{1}$ \\
\hline IMC & $26,23(20,61-40,07)^{2}$ & $26,68(15,29-36,66)^{2}$ & $25,23(16,39-34,38)^{3}$ & $26,54(17,73-36,64)^{3}$ \\
\hline \multicolumn{5}{|c|}{ Bioimpedância elétrica } \\
\hline$\% G C T$ & $31,5(20,2-45)^{1}$ & $37,2(16,2-47,6)^{1}$ & $21,1(7,2-33,8)^{1}$ & $27,5(16-36,3)^{1}$ \\
\hline Resistência & $579,9(467-781)^{4}$ & $580,9(447-799)^{4}$ & $497,2(377-687)^{5}$ & $485,4(343-657)^{5}$ \\
\hline Reactância & $66,2(43-84)^{1}$ & $57,5(32-85)^{1}$ & $65,1(49-85)^{1}$ & $53,5(36-70)^{1}$ \\
\hline Ângulo de fase & $6,5(4,8-8,4)^{1}$ & $5,6(3,6-7,7)^{1}$ & $7,5(5,9-9,5)^{1}$ & $6,3(4,3-8,2)^{1}$ \\
\hline \multicolumn{5}{|c|}{ Tomografia computadorizada } \\
\hline GV & $71,8(11,2-202,7)^{1}$ & $120,2(9,1-231,2)^{1}$ & $95,6(13,4-295,8)^{1}$ & $158,1(9,9-379,6)^{1}$ \\
\hline
\end{tabular}

IMC: índice de massa corporal; \%GCT: percentual de gordura corporal total; GV: gordura visceral; ${ }^{1} p=0,000 ;{ }^{2} p=0,627 ;{ }^{3} p=0,121 ;{ }^{4} p=0,942 ;{ }^{5} p=0,387$. 
$\mathrm{Na}$ investigação sobre a relação entre $\mathrm{AF}$ e $\mathrm{GV}$, a tabela 2 evidencia que os valores médios do $\mathrm{AF}$ não diferem entre os indivíduos com e sem excesso de GV, exceto para as mulheres adultas.

Conforme demonstra a tabela 3 , os coeficientes de correlação entre a área de GV e o \%GCT foram estatisticamente significantes para ambos os sexos e grupos etários, sendo o grupo da mulher adulta o que apresentou a correlação mais forte. Observam-se baixas correlações entre a área de GV e o AF, sem significância estatística.

Conforme os dados da tabela 4, a BIA apresentou pontos de corte de maior sensibilidade do que especificidade para a identificação de uma área de GV considerada de risco para todos os grupos avaliados, exceto para o grupo das idosas. Utilizando o ponto de corte para excesso de $\mathrm{GV}\left(\geq 130 \mathrm{~cm}^{2}\right)$, identificado pela TC, as áreas sob a Curva ROC foram elevadas e maiores para os indivíduos adultos comparadas com as dos idosos.

Tabela 2. Valores médios, mínimos e máximos do AF-BIA, segundo as áreas de GV-TC, por sexo e grupo etário

\begin{tabular}{lccccc}
\hline & \multicolumn{2}{c}{ Feminino } & & \multicolumn{2}{c}{ Masculino } \\
\cline { 2 - 3 } \cline { 5 - 6 } & Adulto $^{\mathbf{1}}$ & Idoso $^{\mathbf{2}}$ & & Adulto $^{\mathbf{2}}$ & Idoso $^{2}$ \\
\hline $\mathrm{GV} \geq$ & 6,32 & 5,67 & & 7,56 & 6,41 \\
$130 \mathrm{~cm}^{2}$ & $(5,45-6,77)$ & $(4,34-6,93)$ & & $(5,91-8,57)$ & $(4,34-8,29)$ \\
$\mathrm{GV}<$ & 6,59 & 5,70 & & 7,52 & 6,28 \\
$130 \mathrm{~cm}^{2}$ & $(4,85-8,46)$ & $(3,68-7,72)$ & & $(6,01-9,55)$ & $(4,33-7,74)$ \\
\hline
\end{tabular}

AF-BIA: ângulo de fase medido pela bioimpedância; GV-TC: gordura visceral medida pela tomografia computadorizada; ${ }^{1} p<0,05 ;{ }^{2} p \geq 0,05$.

Tabela 3. Coeficientes de correlação entre a área de GV determinada pela TC com 0 \%GCT e o AF obtidos pela BIA, segundo sexo e grupo etário

\begin{tabular}{llllll}
\hline & \multicolumn{2}{c}{ Feminino } & & \multicolumn{2}{c}{ Masculino } \\
\cline { 2 - 3 } \cline { 5 - 6 } & Adulto & Idoso & & Adulto & Idoso \\
\hline GV $\times \%$ GCT & $0,611^{1}$ & $0,579^{1}$ & & $0,597^{1}$ & $0,438^{2}$ \\
GV $\times$ AF & $-0,16^{3}$ & $0,144^{3}$ & & $0,100^{3}$ & $0,104^{3}$ \\
\hline
\end{tabular}

GV: gordura visceral; \%GCT: percentual de gordura corporal total, AF: ângulo de fase; ${ }^{1} p=$ $0,000,{ }^{2} p=0,003,{ }^{3} p>0,05$.

Tabela 4. Pontos de corte e áreas sob a Curva ROC, sensibilidade e especificidade do melhor ponto de corte para $0 \% \mathrm{GCT}$ identificado pela BIA para determinar excesso de GV, segundo sexo e grupo etário

\begin{tabular}{|c|c|c|c|c|}
\hline & \multicolumn{2}{|c|}{ Feminino } & \multicolumn{2}{|c|}{ Masculino } \\
\hline & Adulto & Idoso & Adulto & Idoso \\
\hline Ponto de corte \%GCT & 34,30 & 34,80 & 21,5 & 24,25 \\
\hline Área sob a Curva ROC & 0,845 & 0,711 & 0,808 & 0,694 \\
\hline Sensibilidade (\%) & 83,3 & 66,7 & 78,6 & 82,1 \\
\hline Especificidade (\%) & 79,1 & 69 & 70,6 & 62,5 \\
\hline
\end{tabular}

\%GCT: percentual de gordura corporal total.

\section{DISCUSSÃO}

Este estudo avaliou a estimativa da gordura visceral por meio do percentual de gordura corporal total, dado pela BIA, utilizando como método de referência a tomografia computadorizada, em adultos e idosos, e investigou as diferenças existentes entre esses grupos etários e sexo. Trata-se de um estudo de cunho exploratório, baseado em pesquisas publicadas anteriormente que recomendam a BIA como um bom indicador de GV (15), porém que não utilizam como referência métodos considerados padrão-ouro, como a TC.

Sabe-se que a TC é o método mais confiável para quantificar a GV, entretanto tem suas limitações (4), conforme já mencionado neste trabalho. Em relação à BIA, é um método que está se popularizando, sendo citado frequentemente como método confiável para a estimativa de gordura corporal (5). Contudo, poucos são os estudos publicados que aplicaram a BIA para indicação da adiposidade visceral.

Em nossos achados, as mulheres apresentaram valores médios de \%GCT superiores aos dos homens, nos dois grupos etários. Esses resultados são compatíveis com os achados em outras pesquisas, tanto para indivíduos de 60 a 81 anos quanto naqueles pertencentes a outras faixas etárias (16-18).

Esse percentual de gordura, quantificado no presente estudo por meio da BIA, permitiu ainda observar que as mulheres idosas apresentaram valores maiores que as adultas. Em concordância, França e Barbosa (18), analisando o perfil da composição corporal de mulheres praticantes de atividade física regular em diferentes faixas etárias, entre 23 e 78 anos, observaram que mesmo na ausência de aumento de peso corporal houve aumento na quantidade de gordura corporal que acompanhou o envelhecimento.

É atualmente descrita com frequência a relação entre gordura abdominal e morbidades, demonstrando a necessidade de estudos que desenvolvam métodos simples e acessíveis para a avaliação da composição corporal, principalmente do idoso, e, em especial, para diagnosticar a GV, já que esta apresenta estreita relação com as doenças metabólicas, sobretudo as cardiovasculares $(19,20)$.

Os valores médios de GV, assim como o excesso desse tecido $\left(\geq 130 \mathrm{~cm}^{2}\right)$, foram maiores nos indivíduos idosos e, em especial, nos homens. Nos indivíduos idosos, existe maior acúmulo de gordura abdominal, principalmente da GV, quando comparados com os adultos, e isso ocorre para ambos os sexos, principalmente nos homens. Percebe-se que existe um maior acúmulo de 
GV na região abdominal, e, nas mulheres, o acúmulo de gordura ocorre principalmente na região gluteofemoral e no tecido abdominal subcutâneo, o que explica os resultados supracitados (18,21-24).

No presente estudo, os pontos de corte do \%GCT nos homens para predizer GV em adultos e idosos (21, $5 \%$ e $24,25 \%$, respectivamente) foram menores do que o preconizado pela literatura para definir risco para doenças crônicas (25\%) (25). O inverso ocorreu com as mulheres que apresentaram $34,3 \%$ e $34,8 \%$ para adultas e idosas, respectivamente, versus o ponto de corte de $30 \%$ preconizado pela literatura (25). Ocorre que os homens possuem maior quantidade de gordura abdominal e, consequentemente, menor ponto de corte da gordura corporal total para predizer GV. Quando a gordura abdominal não é a predominante, como é o caso das mulheres, é necessária uma maior quantidade da gordura corporal total para poder predizer GV, elevando, assim, o ponto de corte observado nesse estudo. Isso possivelmente foi encontrado, também, pelo modelo tradicional de BIA utilizado neste estudo, que estima a quantidade total de gordura corporal, não diferenciando a gordura da região abdominal dos outros segmentos corporais.

O AF é um parâmetro obtido diretamente dos valores de resistência e reactância e pode ser associado à morte celular ou à alteração na permeabilidade seletiva da membrana celular (16,26-29). Assim, é esperado que ocorram mudanças em seus valores de acordo com o sexo e envelhecimento (30). Em concordância a essas observações, neste estudo encontraram-se valores médios de reactância e AF menores entre as mulheres e os idosos de ambos os sexos. A justificativa, de acordo com Lima e cols. (20), reside na diminuição da massa muscular relacionada ao envelhecimento e, como característica natural feminina, observa-se menor quantidade de massa magra em comparação à do sexo masculino.

Diversos estudos realizados com pacientes portadores de algumas patologias mostraram boa correlação entre $o$ AF e o tempo de sobrevida, o índice de morbidade e as complicações pós-operatórias (30-35). Com base nessas evidências, buscou-se, neste estudo, explorar os valores do AF nos indivíduos com e sem excesso de GV e sua correlação com essa gordura como indicador de morbidades. Entretanto, nessas análises não houve diferença significativa nos valores do AF para indivíduos com e sem excesso de GV, e, ainda, foram observadas fracas correlações.

Assim, novamente chamamos a atenção para o modelo de BIA utilizado para avaliar a gordura abdominal nesta pesquisa, que possui vantagens como praticida- de, rapidez e custo relativamente baixo, porém para o objetivo em questão o método da BIA segmentar talvez possibilitasse uma maior precisão na avaliação da gordura abdominal, tornando-se o método mais indicado. Segundo Howell (36), o organismo não é um condutor uniforme e de comprimento constante, assemelha-se a um conjunto de cinco cilindros separados: dois braços, duas pernas e tronco. Coerente com essa teoria, o modelo da BIA segmentar possui quatro pares de eletrodos, um para cada um dos membros superiores e inferiores, possibilitando, assim, a análise corporal por compartimentos. A partir desse método, verifica-se com maior acurácia a massa gorda total e por segmento (36). Dessa forma, a análise da gordura do tronco permitirá, possivelmente, estimar com maior precisão a GV.

Já são discutidas na literatura novas técnicas de referência para avaliar a GV por meio da BIA, posicionando os eletrodos diretamente no abdome. Segundo os autores, esse método permite avaliar com mais precisão a gordura abdominal, incluindo a visceral $(7,37,38)$. Como exemplo, o estudo de Nagai e cols. (7) concluiu que esse é um método simples e conveniente para a estimativa da GV nos indivíduos estudados, com forte correlação $(r=0,920)$ entre a GV identificada pela TC e a determinada pela BIA.

Em outro estudo, Watson e cols. (37), seguindo a metodologia proposta por Ryo e cols. (38), ratificaram a importância da BIA abdominal. Os autores utilizaram essa técnica combinada à medida da circunferência da cintura, tendo como referência a GV estimada pela TC, e demonstraram elevada correlação $(0,904)$ entre a BIA abdominal e a GV.

Em conclusão, a BIA apresentou satisfatória sensibilidade e especificidade para predizer gordura visceral nos dois grupos etários, sendo que os pontos de corte que identificaram uma área de risco foram superiores nas mulheres adultas. Novos estudos tornam-se necessários utilizando outros aparelhos, a exemplo da BIA segmentar assim como outras técnicas de posicionamento dos eletrodos com o intuito de melhorar essa predição, especialmente em indivíduos idosos pela provável influência de fatores relacionados ao envelhecimento.

Agradecimentos: ao Conselho Nacional de Desenvolvimento Científico e Tecnológico (CNPq), que financiou esta pesquisa (processo no $470882 / 2006-9$ ).

Declaração: os autores declaram não haver conflitos de interesse científico neste estudo. 


\section{REFERÊNCIAS}

1. Kissebah $\mathrm{AH}$, Vydelingun $\mathrm{N}$, Murray R, Evans DJ, Hartz AJ, Kalkhoff RK. Relation of body fat distribution to metabolic complication of obesity. J Clin Endocrinol Metab. 1982;54:254-60.

2. Leite CC, Matsuda D, Wajchenberg BJ, Cerri GG, Halpern A. Correlação da medida de espessura intra-abdominal medida pela ultra-sonografia com os fatores de risco cardiovascular. Arq Bras Endocrinol Metab. 2000;44(1):49-55.

3. Sjöström L, Kvist H, Cedderblad A, Tylén U. Determination of total adipose tissue and body fat in women by computed tomography 40K, and tritium. Am J Physiol. 1986;250:736-45.

4. Van Der Kooy K, Leenen R, Seidell JC, Deurenberg P, Visser M. Abdominal diameters as indicators of visceral fat: comparison between magnetic resonance imaging and anthropometry. $\mathrm{Br} \mathrm{J}$ Nutr. 1993;70:47-58.

5. Britto EP, Mesquita ET. Bioimpedância elétrica aplicada à insuficiência cardíaca. Rev SOCERJ. 2008;21(3):178-83.

6. Vieira ACR, Alvarez MM, Marins VMR de, Sichieri R, Veiga GV. Desempenho de pontos de corte do índice de massa corporal de diferentes referências na predição de gordura corporal em adolescentes. Cad Saúde Pública. 2006;22(8):1681-90.

7. Nagai M, Komiya H, Mori Y, Otha T, Kasahara Y, Ikeda Y. Development of a new method for estimating visceral fat area with multi frequency bioelectrical impedance. J Exp Med. 2008;214:105-12.

8. Roriz AKC, Oliveira CC, Moreira PA, Medeiros JMB, Sampaio LR. Methods of predicting visceral fat in Brazilian adults and older adults: a comparison between anthropometry and computadorized tomografy. Arch Latinoam Nutr. 2011;61(1):5-12.

9. Sant'anna MSL, Tinoco ALA, Rosado LEFPL, Sant'anna LFR, Mello AC, Brito, et al. Body fat assessment by bioelectrical impedance and its correlation with different anatomical sites used in the measurement of waist circumference in children. J Pediatr. 2009;85(1):61-6.

10. Slinde F, Rossander-Hulthen L. Bioelectrical impedance: effect of three identical meals on diurnal impedance variation and calculation of body composition. Am J Clin Nutr. 2001;74:474-8.

11. Coppini LZ, Bottoni A, Teixeira Silva ML. Aplicação da análise da impedância bioelétrica na avaliação nutricional. Rev Bras Nutr Clin. 1998;13:81-9.

12. Baumgartner RN, Chumlea WC, Roche AF. Bioelectric impedance phase angle and body composition. Am J Clin Nutr. 1988;48:16-23.

13. Seidell JC, Oosterlee A, Thijssen MAO, Burema J. Assessment of intra-abdominal and subcutaneous abdominal fat: relation between anthropometry and computed tomography. Am J Clin Nutr. 1987;45:7-13.

14. Desprès JP, Lamarche B. Effects of diet and physical activity on adiposity and body fat distribution: implications for the prevention of cardiovascular disease. Nutr Res Rev. 1993;6:137-59.

15. Fernandez RA, Rosa CSC, Buonani C, Oliveira AR, Freitas Junior IF. Utilização da impedância bioelétrica na indicação do excesso de gordura visceral e subcutânea. J Pediatr. 2007;83(6):529-34.

16. Rech CR, Cordeiro BA, Petroski EL, Vasconcelos FAG. Validation of bioelectrical impedance for the prediction of fat-free mass in Brazilian elderly subjects. Arq Bras Endocrinol Metab. 2008;52(7):1163-71.

17. Correa FHS, Taboada GF, Junior CRMA, Faria AM, Clemente ELS, Fuks AG, et al. Influência da gordura corporal no controle clínico e metabólico de pacientes com diabetes mellitus tipo 2. Arq Bras Endocrinol Metab. 2003;47(1):62-8.

18. França AM, Barbosa MTS. Perfil da composição corporal de muIheres de diferentes faixas etárias praticantes de atividade física regular. Rev Dig Educ Fis. 2007;2(1):1-13.

19. Sampaio LR. Avaliação nutricional e envelhecimento. Rev Nutr. 2004;17(4):507-14.
20. Lima LRA, Rech CR, Petroski EL. Utilização da impedância biolelétrica para estimativa da massa muscular esquelética em homens idosos. Arch Latinoam Nutr. 2008;58(4):386-91.

21. Enzi G, Sergi G, Bussolotto M, Ceccon A, Giantin V, Beninca P. Methods for quantitation of body composition, with particular reference to lean body mass. In Amino Acid and Protein Metabolism in Health and Disease. Bedford, Great Britain: Smith-Gordon; 1997. p. 5-11.

22. Perissinotto E, Pisent C, Sergi G, Grigoletto F, Enzi G. Anthropometric measurements in the elderly: age and gender differences. Br J Nutr. 2002;87:177-86.

23. Kim JA, Choi CJ, Yun KS. Cut-off values of visceral fat area and waist circumference: diagnostic criteria for abdominal obesity in a Korean population. J Korean Med Sci. 2006;21(6):1048-53.

24. Sampaio LR, Simões EJ, Assis AMO, Ramos LR. Validity and reliability of the sagittal abdominal diameter as a predictor of visceral abdominal fat. Arq Bras Endocrinol Metab. 2007;51:980-6.

25. American Dietetic Association. Position of the American Dietetic Association and the Canadian Dietetic Association: nutrition for physical fitness and athletic performance for adults. J Am Diet Assoc. 1993;93(6):691-6.

26. Eickemberg M, Oliveira CC, Roriz AKC, Sampaio LR. Bioimpedância elétrica e sua aplicação em avaliação nutricional. Rev Nutr. 2011;24(6):883-93.

27. Negano M, Suita S, Fukuoke TY. The validity of bioelectrical impedance phase angle for nutritional assessment in children. $J$ Pedriatr Surg. 2000;35:1035-9.

28. Silva LMDL, Caruso L, Martini LA. Aplicação do ângulo de fase em situações clínicas. Rev Bras Nutr Clin. 2007;22(4):317-21.

29. Barbosa-Silva MC, Barros AJ, Post CL, Waitzberg DL, Heymsfield SB. Can bioelectrical impedance analysis identify malnutrition in preoperative nutrition assessment? Nutrition. 2003;19(5):422-6.

30. Barbosa-Silva MCG, Barros AJD. Bioelectrical impedance and individual characteristics as prognostic factors for post-operative complications. Clin Nutr. 2005;24(5):830-8.

31. Guida B, De Nicola L, Pecorato P, Trio R, Di Paola F, lodice C, et al. Abnormalities of bioimpedance measures in overweight and obese hemodialyzed patients. Int J Obes. 2001;25:265-72.

32. Gupta D, Lis CG, Dahlk SL, Vashi PG, Grutsch JF, Lammersfeld CA. Bioelectrical impedance phase angle as a prognostic indicator in advanced pancreatic cancer. Br J Nutr. 2004;92:957-62.

33. Selberg O, Selberg D. Norms and correlates of bioimpedance phase angle in healthy human subjects, hospitalized patients, and patients with liver cirrhosis. Eur J Appl Physiol. 2002;86(6):509-16.

34. Avram MM, Sreedhara R, Fein PA, Oo KK, Chattopadhyay J, Mittmam N. Survival of hemodialysis and peritoneal dialysis over 12 years with emphasis on nutritional parameters. Am J Kidney Dis. 2001;37:S77-S80.

35. Fein PA, Gundumalla G, Jorden A, Matza B, Chattopadhyay J, Avram MM. Usefullness of bioelectrical impedance analysis in monitoring nutrition status and survival in peritoneal dialysis patients. Adv Perit Dial. 2002;18:195-9.

36. Howell W. Anthropometry and Body Composition Analysis. In: Matarese L, Gottschlich M. Contemporary Nutrition Support Practice. A Clinical Guide. W.B. Saunders; 1998. p. 33-46.

37. Watson S, Blundell HL, Evans WD, Griffiths $H$, Newcombe RG, Rees DA. Can abdominal bioelectrical impedance refine the determination of visceral fat from waist circumference? Physiol Meas. 2009;30:N53-8.

38. Ryo M, Maeda K, Onda T, Katashima M, Okumiya A, Nishida M, et al. A new simple method for the measurement of visceral fat accumulation by bioelectrical impedance. Diabetes Care. 2005;28(2):451-3. 